\title{
Symmetropy, an entropy-like measure of visual symmetry
}

\author{
EIJI YODOGAWA \\ Musashino Electrical Communication Laboratory, Nippon Telegraph and Telephone Public Corporation \\ Musashino-shi, Tokyo, Japan
}

\begin{abstract}
A new objective measure of symmetry for single patterns, called symmetropy, is developed on two bases, the two-dimensional discrete Walsh transform of a pattern and the entropy concept in information theory. It is extended to a more general measure, called the symmetropy vector. In order to test the predictive power of the symmetropy vector, multiple regression analyses of judged pattern goodness and of judged pattern complexity were carried out. The analyses show that the symmetropy vector predicts pattern goodness and pattern complexity, as well as the amount of symmetry in a pattern. They also suggest that pattern goodness is a concept based on the holistic properties of a pattern, while pattern complexity (or simplicity) is a concept based on both holistic and partial properties of a pattern.
\end{abstract}

According to the Gestalt organizational laws of visual perception, we usually see a visual pattern as the simplest organization of all possible organizations from the elements in the visual field (Koffka, 1935). There can be no doubt that the Gestalt laws, such as proximity, similarity, continuation, symmetry, etc., work well in an intuitive sense. However, an adequate means for specifying objective variables underlying the organizations predicted by the laws is lacking. To remedy this lack, recent studies have turned to quantitative approaches to pattern "goodness," which has been regarded as one of the most important Gestalt concepts of figural organizations.

Attneave $(1954,1955)$ demonstrated that many of the Gestalt laws constitute redundancy in visual stimulation, and may be quantified within a framework of information theory. He investigated the symmetry effect on memory for patterns and found that symmetrical patterns were more accurately reproduced from memory only when they contained less information than asymmetrical patterns. A similar approach was taken by Hochberg and McAlister (1953).

In these studies, the redundancy concept was regarded as a measurable property for single patterns and was considered directly related to the goodness of the patterns. On the other hand, Garner (1962) pointed out that redundancy was a property of sets of

\footnotetext{
The author would like to thank K. Noda of Tokyo University of Agriculture and Technology and N. Kuroyanagi for their encouragement and support in this research, and S. Hashimoto and I. Masuda for their useful discussions and suggestions. He would also like to thank S. Imai of Hokkaido University, who provided many helpful comments and recommended writing this manuscript. The author's mailing address is: Musashino Electrical Communication Laboratory, NTT, 3-9-11, Midori-cho, Musashinoshi, Tokyo 180, Japan.
}

patterns, not of single patterns, and suggested that each single pattern was perceived not only as that one pattern, but also as one of a subset of equivalent patterns. Furthermore, he hypothesized that pattern goodness was inversely related to the size of this inferred subset of equivalent patterns. Later, Garner and Clement (1963) obtained experimental results supporting this hypothesis. In their experiment, two patterns are said to be equivalent if one pattern is produced by reflections and 90 -deg rotations of the other. Although Garner directly related pattern goodness to the size of equivalent subsets, Imai et al. (1976) related it to the intrapattern transformation structures, rather than the subset size. The transformation structures of patterns were defined by invariant properties for three cognitive transformations: mirroring on a diagonal axis, mirroring on the vertical axis, and 180-deg rotation. Their experimental results showed that pattern goodness judgments were dependent on the transformation structures. In addition, Howe (1980) investigated the effect of partial symmetry on pattern goodness judgments and found that judged pattern goodness was a function not simply of equivalent subset size, but also of amount of partial symmetry within patterns that were asymmetrical when considered as wholes. Szilagyi and Baird (1977) investigated the quantitative relationship between pattern goodness and the amount of symmetry in visual designs, and showed that pattern goodness was inversely related to the quantitative degree of asymmetry. It should, however, be noted that the goodness of a pattern was operationally defined by the percent of subjects who created such a pattern on the basis of aesthetic preference.

These studies suggest the importance of symmetry in pattern goodness. Furthermore, symmetry has been regarded as a fundamental determinant of judged 
pattern complexity (Chipman, 1977). It would be useful, therefore, to develop a quantitative and objective measure of symmetry in order to predict perceptual organization. Despite the importance of symmetry, there has been little work to date on measures of symmetry, and quantitative and objective measures proposed by previous studies have several problems. First, in most studies, the amount of symmetry is measured by the number of axes through which reflection will reproduce the same pattern, or by the size of an equivalent subset, as Garner and Clement suggested. However, symmetry is a continuum. Second, most measures of symmetry are not adequate to measure the amount of partial symmetry within a pattern. Third, the measures do not have sufficient predictive power. (Zusne, 1965, 1971, devised three single-form measures of symmetry - third moment of area, maximum self-overlap, and asymmetry of coordinates-but none of these measures was a good predictor of symmetry judgments.)

The present paper develops a new objective physical measure of visual symmetry that solves these problems. First, we consider the relationship between the amount of symmetry in a single pattern and the concept of entropy in information theory. Second, an entropy-like measure, called symmetropy, is proposed as an objective physical measure of symmetry. Then it is extended to a more general measure, called the symmetropy vector. Third, multiple regression analyses are carried out to test the utility of the symmetropy vectors as predictors of pattern goodness and complexity. Finally, some of the advantages of symmetropy vectors are discussed. A distinction between pattern goodness and pattern complexity (or simplicity) is also discussed on the basis of the results of the regression analyses.

\section{AMOUNT OF SYMMETRY AND THE ENTROPY CONCEPT}

The concept of redundancy in information theory has been used to quantify the amount of symmetry and pattern goodness, but without much success. This paper reports an attempt to apply the entropy concept to quantification of the amount of symmetry in a pattern.

In information theory, an information-generating mechanism is described by an information source. The source is emitting a sequence of symbols from a fixed finite source alphabet. Successive symbols occur according to some fixed probability law. Thus, an information source is specified by giving a set of finite symbols, or a source alphabet, and the probabilities with which the symbols occur. The entropy of an information source is defined to be the average amount of information per source symbol. Symmetry may be generally considered as a form of re- dundancy. Since entropy, or its inverse, redundancy, is measurable in bits of information, so is symmetry, if an entropy-like measure of symmetry can be found. However, as Garner (1962) pointed out, there is inconsistency in using the redundancy concept with respect to single patterns, since redundancy is a property of sets of patterns. In this paper, this inconsistency is solved by the use of an orthogonal transformation of single patterns.

In the area of signal processing, the Fourier transform of an original signal is often used as the primary method of analysis, since the Fourier transform makes it easier to understand the nature of the signal. In a similar way, an original pattern may be transformed into some pattern in another mathematical space, which is convenient for analyzing the amount of symmetry in the original pattern. The two-dimensional Walsh transform is one such transform.

Two-dimensional Walsh functions are particularly suited to the present purpose. They form a complete orthonormal set, which is equally divided into four subsets according to the types of symmetry-vertical, horizontal, 180-deg rotational, and double (i.e., both vertical and horizontal. Note that the doubly symmetric patterns also have rotational symmetry. Although Fourier (sine-cosine) functions also have this property, they do not have the following property: The two-dimensional Walsh functions take only two values, +1 and -1 . This property facilitates the com-

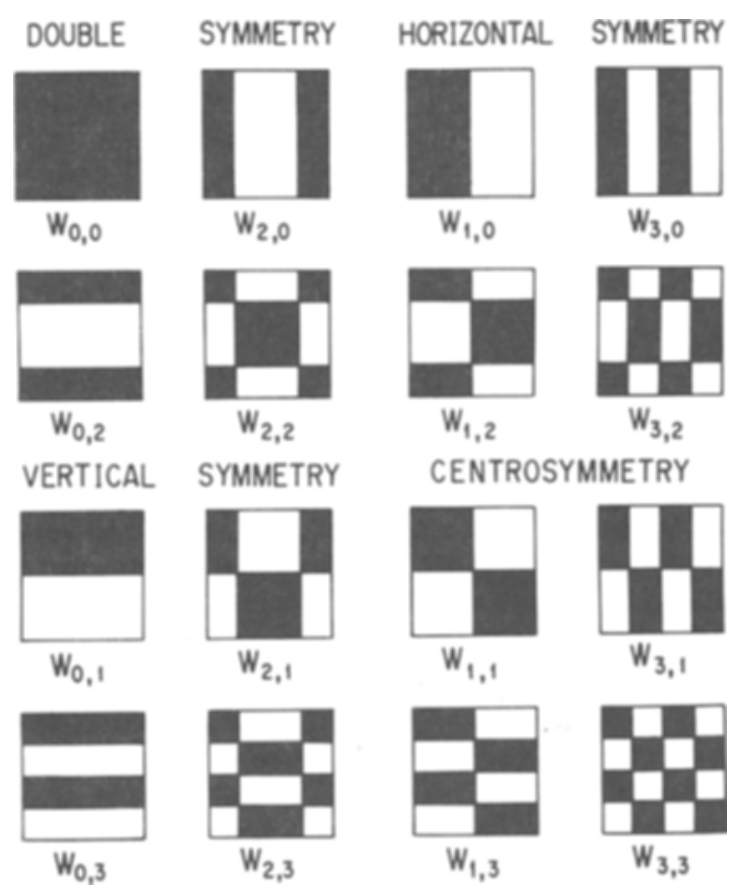

Figure 1. Two-dimensional Walsh functions (for $N=4$ ) arranged with respect to the types of symmetry described in the text. Black represents +1 and white represents -1 . 
putation of the two-dimensional Walsh transform of patterns.

The first 16 of the two-dimensional Walsh functions are shown in Figure 1. Thus, the two-dimensional Walsh transform, which is defined in the next section, decomposes any single pattern into four components of symmetry. Here, we can think of a pattern as a zero-memory information source ${ }^{1}$ with source alphabet $\left(s_{1}, s_{2}, s_{3}, s_{4}\right.$-the four types of symmetry) and with the probability of $s_{i}$ equal to the normalized value $P_{i}$ of the corresponding component. Then we can think of the entropy of the source as a measure of symmetry in the pattern, or the source (Abramson, 1963). In other words, if the value of a certain component of the four types of symmetry is larger than the values of other components, the original pattern is richer in symmetry. On the other hand, if the values of the four components are almost equal, the original pattern is poor in symmetry. This notion is formalized in the following section.

\section{FORMULATION OF AN ENTROPY-LIKE MEASURE OF SYMMETRY}

\section{Two-Dimensional Discrete Walsh Transforms}

In the Appendix, Walsh functions are explicitly defined, and their fundamental properties, a brief history, the advantages they have over Fourier functions, and their applications are presented. A reader who is unfamiliar with Walsh functions should refer to this first in order to understand the following definitions.

The two-dimensional discrete Walsh function $W_{m, n}(i, j)$ of order $(m, n)$ and argument $(i, j)$ can be defined as

$$
\begin{gathered}
w_{m, n}(i, j)=w_{n}(i) w_{m}(j), \\
m, n, i, j=0,1,2, \ldots, N-1,
\end{gathered}
$$

where $\mathrm{N}=2^{\mathrm{q}}$ ( $q$ is a positive integer), and $\mathrm{w}_{n}(\mathrm{i})$ and $\mathrm{w}_{\mathrm{m}}(\mathrm{j})$ are the one-dimensional discrete Walsh functions defined in the Appendix.

Here, we define the two-dimensional discrete Walsh functions on a square region, which is divided into $\mathbf{N} \times \mathbf{N}$ equal square subregions called cells (see Figure 1). These functions can be represented in matrix form as $\left[W_{m, n}(i, j)\right]$, where $W_{m, n}(i, j)$ is the value of the $(m, n)^{\text {th }}$ order Walsh function in the $i^{\text {th }}$ row cell in the $j^{\text {th }}$ column.

Patterns used in this paper are restricted to square matrices, each consisting of $\mathrm{N} \times \mathrm{N}$ square cells, where $\mathrm{N}=2^{q}$ ( $q$ is a positive integer). The use of the twodimensional discrete Walsh transform requires that $\mathrm{N}$ be equal to a power of two. This requirement, however, is not a limitation, since any pattern can be embedded in an $\mathbf{N} \times \mathbf{N}$ matrix. A pattern can be written as $\left[x_{i, j}\right]$, where $x_{i, j}$ is the value of gray level in the $i^{\text {th }}$ row cell in the $j^{\text {th }}$ column and $i, j=0,1, \ldots$, $\mathrm{N}-1$. In particular, if there are just two gray levels, "black" and "white," we usually represent them by 1 and 0.

By making use of the above definitions, the twodimensional discrete Walsh transform of a pattern, $\left[x_{i, j}\right]$, of $\mathbf{N} \times \mathbf{N}$ cells, is given by the expression

$$
\begin{gathered}
a_{m, n}=\frac{1}{N^{2}} \sum_{i=0}^{N-1} \sum_{j=0}^{N-1} x_{i, j} w_{m, n}(i, j), \\
m, n=0,1,2, \ldots, N-1,
\end{gathered}
$$

and the inverse transformation is given by

$$
\begin{gathered}
x_{i, j}=\sum_{m=0}^{N-1} \sum_{n=0}^{N-1} a_{m, n} w_{m, n}(i, j), \\
i, j=0,1,2, \ldots, N-1 .
\end{gathered}
$$

We will call $a_{m, n}$ the two-dimensional Walsh spectrum, and $\left(a_{m, n}\right)^{2}$ the two-dimensional Walsh power spectrum. From Equation 1, for $m, n=0$, we obtain

$$
a_{0,0}=\frac{1}{N^{2}} \sum_{i=0}^{N-1} \sum_{j=0}^{N-1} x_{i, j}
$$

Therefore, $a_{0,0}$ is interpreted as the average value for the summation of gray levels in the pattern $\left[x_{i, j}\right]$.

Using the two-dimensional discrete Walsh transform, a pattern $\left[x_{i, j}\right]$ is transformed from a representation in the original domain (or the image domain) to its corresponding representation in the sequence domain, which is the counterpart of the frequency domain in the discrete Fourier transform.

The two-dimensional Walsh power spectra $\left(a_{m, n}\right)^{2}$ can be normalized as follows:

$$
\begin{gathered}
K=\sum_{m=0}^{N-1} \sum_{n=0}^{N-1}\left(a_{m, n}\right)^{2}-\left(a_{0,0}\right)^{2}, \\
p_{m, n}=\left(a_{m, n}\right)^{2} / K,
\end{gathered}
$$

where we assume that $K \neq 0$. Thus, we obtain $p_{m, n} \geqslant 0$ and

$$
\sum_{m, n}^{N-1} p_{m, n}=1,
$$

where the sum is taken over all ordered pairs $(m, n)$ except $(0,0)$ for $0 \leqslant m, n \leqslant N-1$. The reason for 
subtracting $\left(a_{0,0}\right)^{2}$ from the summation of the Walsh power spectra in Equation 2 is that $\left(a_{0,0}\right)^{2}$ is the Walsh power spectrum of the pattern $\left[x_{i, j}\right]$ for $W_{0,0}(i, j)$, which provides no shape information. The normalized Walsh power spectra $\mathrm{p}_{\mathrm{m}, \mathrm{n}}$ defined in Equation 3 will be used in the next section.

\section{A New Measure of Symmetry}

To develop a new measure of symmetry in a pattern, we will use the following property of the twodimensional Walsh functions with respect to symmetry:

$$
\mathrm{w}_{\mathrm{m}, \mathrm{n}}(\mathrm{i}, \mathrm{j})= \begin{cases}\text { vertically symmetric, } & \text { if } \mathrm{m}=\text { even and } \mathrm{n}=\text { odd; } \\ \text { horizontally symmetric, } & \text { if } \mathrm{m}=\text { odd and } \mathrm{n}=\text { even; } \\ \text { centrosymmetric, } & \text { if } \mathrm{m}, \mathrm{n}=\text { odd; } \\ \text { doubly symmetric, } & \text { if } \mathrm{m}, \mathrm{n}=\text { even. }\end{cases}
$$

This is easily proven from the symmetries of the onedimensional Walsh functions presented in the Appendix. By using this property, a set of two-dimensional Walsh power spectra of a pattern can be easily divided into four components according to the types of symmetry involved, as follows:

vertically symmetric component,

$$
P_{1}=\sum_{\substack{\text { m=even } \\ n=\text { odd }}} p_{m, n} ;
$$

horizontally symmetric component,

$$
\mathbf{P}_{2}=\sum_{\substack{\text { m=odd } \\ n=\text { even }}} \mathrm{p}_{\mathrm{m}, \mathrm{n}}
$$

centrosymmetric component,

$$
P_{3}=\sum_{\substack{\text { m=odd } \\ n=\text { odd }}} p_{m, n} ;
$$

and doubly symmetric component,

$$
\mathrm{P}_{4}=\sum_{\substack{\mathrm{m}=\text { even } \\ \mathrm{n}=\text { even }}} \mathrm{p}_{\mathrm{m}, \mathrm{n}} \text {. }
$$

In each equation, the sum is taken over all ordered pairs $(m, n)$ except $(0,0)$ for $0 \leqslant m, n \leqslant N-1$.

Now we can define a new measure of symmetry. Applying the entropy function in information theory to the four components of the types of symmetry, we define

$$
S_{N}=-\sum_{k=1}^{4} P_{k} \log _{2} P_{k} \text {, }
$$

and we call $\mathrm{S}_{\mathrm{N}}$ the symmetropy.

The symmetropy $S_{N}$ can be explained as follows: (1) A pattern can be considered as a zero-memory information source consisting of the four types of symmetry, each occurring with a probability whose value is equal to the corresponding symmetric component $P_{k}(k=1,2,3,4)$. (2) Each symmetric component $P_{k}$ is given as the sum of the two-dimensional normalized Walsh power spectra of the pattern for the same symmetry type. (3) The symmetropy $S_{N}$ means the entropy of such an information source, and can be considered as a quantitative and objective measure of symmetry.

Whether or not the human visual system actually performs such computations as those for entropy and two-dimensional Walsh power spectra remains conjectural.

We can easily verify the following properties of the symmetropy. The symmetropy is invariant under the following operations on patterns: (1) 90-deg rotations, (2) dilations or contractions by $2 \mathrm{k}(\mathrm{k}$ is a nonzero integer) times in the horizontal or vertical direction, and (3) black-white reversals of binary patterns (i.e., figure-ground reversals).

The symmetropy of that pattern, which is coincident with one of the two-dimensional Walsh functions, except for the correspondence of each gray level, equals zero. Furthermore, the symmetropy of a doubly symmetric pattern is also zero. In general, all of the patterns that have, for example, perfect vertical symmetry do not necessarily have the same value for their vertically symmetric components because vertically symmetric patterns usually have not only the vertically symmetric, but also the doubly symmetric components. The same goes for horizontally symmetric patterns and centrosymmetric patterns. Therefore, the value of the vertically symmetric component measured in this paper does not perfectly correspond to the direct measurements of the amount of mirroring around a vertical axis. However, it should be noted that if the pattern being examined is identical to one of the pattern templates of the twodimensional Walsh functions, they have perfect correspondence.

\section{Symmetropy Vector}

The symmetropy is defined only for the $2^{q} \times 2^{q}$ patterns. For any $M \times M\left(M \geqslant 3\right.$ and $\left.M \neq 2^{q}\right)$ pattern $A$, we transform the pattern $A$ into the $2^{q} \times 2^{q}$ pattern $\mathrm{B}$, as follows: 
(1) If $M$ is even, let $q$ be a minimum integer which satisfies $2^{q}>M$. Then, pattern $B$ is defined as

$$
\left.\mathbf{B}=\left[\begin{array}{lll}
0 & 0 & 0 \\
0 & \mathrm{~A} & 0 \\
0 & 0 & 0
\end{array}\right]\right)^{2^{q}},
$$

where 0 denotes a zero matrix.

(2) If $M$ is odd, let $q$ be a minimum integer which satisfies $2^{q}>2 M$. Then pattern $B$ is defined as

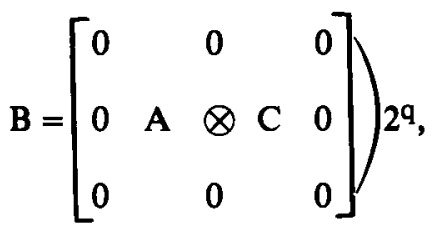

where $0=$ a zero matrix, $C=\left[\begin{array}{ll}1 & 1 \\ 1 & 1\end{array}\right]$, and $\otimes$ denotes the direct, or Kronecker, product. The Kronecker product $A \otimes C$ means replacing each element $a$ in matrix $A$ by the matrix $\left[\begin{array}{ll}a & a \\ a & a\end{array}\right]$. In other words, $A \otimes C$ is the pattern which is formed from dilating pattern $\mathbf{A}$ four times.

In the previous section, we showed that the symmetropy is invariant under dilations of a pattern $2^{k}$ times in the horizontal or vertical direction. Therefore, without loss of generality, the symmetropy of the $\mathbf{M} \times \mathbf{M}$ pattern $\mathrm{A}$ can be defined to be that of the transformed pattern $B$.

In general, the goodness and the complexity of a pattern seem to be directly related not only to the amount of total symmetry but also to the amount of partial symmetry in the pattern (Chipman, 1977; Howe, 1980). Therefore, we now define a measure of partial symmetry and another related measure. Let $A$ be any $M \times M$ pattern, where $2^{r+1} \geqslant M>2^{r}$ and $r$ is a positive integer. To obtain the measure of partial symmetry, some observation windows are introduced. The observation windows are the $2 k \times 2 k$ $(\mathrm{k}=1,2, \ldots, \mathrm{r})$ square regions through which pattern $\mathbf{A}$ is observed at all possible distinct window positions. At each window position in pattern $A$, the symmetropy of each subpattern observed through the $2^{k} \times 2^{k}$ window is computed. Then, the mean symmetropy value, averaged over all possible window positions, and the standard deviation of the symmetropy are computed for each window size. They are denoted by $S_{M}^{k}$ and $o_{M}^{k}$, respectively. Since pattern $A$ is observed through the $2^{k} \times 2^{k}$ window, $\mathrm{S}_{\mathrm{M}}$ can be considered as a measure of partial symmetry. The standard deviations are introduced with the intention of estimating the homogeneity of pattern $\mathbf{A}$ for each window size.
To estimate the amount of symmetry for various observation window sizes, the following vector is introduced.

$$
\mathrm{V}_{\mathrm{M}}=\left(\mathrm{S}_{\mathrm{M}}, \mathrm{S}_{\mathrm{M}}^{\mathrm{r}}, \mathrm{S}_{\mathrm{M}}^{\mathrm{r}-1}, \ldots, \mathrm{S}_{\mathrm{M}}^{1}, \sigma_{\mathrm{M}}^{\mathrm{r}}, \sigma_{\mathrm{M}}^{\mathrm{r}-1}, \ldots, \sigma_{\mathrm{M}}^{1}\right),
$$

where $2^{r+1} \geqslant M>2^{r}$, and $r \geqslant 1$. Vector $V_{M}$ is called the symmetropy vector of the $\mathbf{M} \times \mathbf{M}$ pattern $A$. In addition, $S_{M}, S_{M}^{k}$, and $o_{M}^{k}$ are called the whole symmetropy, the $2^{\mathrm{k}} \times 2^{\mathrm{k}}$ partial symmetropy, and the standard deviation of the $2^{\mathrm{k}} \times 2^{\mathrm{k}}$ partial symmetropy of the $\mathbf{M} \times \mathbf{M}$ pattern $A$, respectively. Here, it should be noted that the symmetropy vector is defined for any $\mathbf{M} \times \mathbf{M}(\mathbf{M} \geqslant 3)$ patterns.

We now consider the relation between the symmetropy vector and the variables that have previously been used in research on the psychophysics of pattern, such as the number of turns, the amount of contour, dispersion, compactness, repetitions, rotations, and several types of symmetry. Since the symmetropy measure has its properties stated in the previous section, it can be said that it is closely related to repetitions, rotations, and several types of symmetry. It seems that the standard deviation of symmetropy reflects dispersion and compactness. Neither the symmetropy measure nor its standard deviation have a clear relation to the number of turns and the amount of contour. However, there seem to be some indirect relations existing between them.

Here, it should be pointed out that the symmetropy vector has been represented in terms of twodimensional Walsh power spectra, and is based on the symmetry concept only, while variables that have been used previously in related studies have been represented in terms of features in the original image domain and are chosen intuitively.

\section{Illustrative Examples}

Consider a $4 \times 4$ pattern, consisting of eight black and eight white cells, as shown in Figure 2A. In pattern $A$, white represents the value 0 and black represents 1 .

The two-dimensional Walsh spectra, obtained from pattern $\mathbf{A}$ by using Equation 1, are given in Table 1.
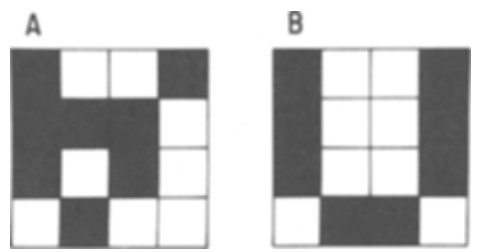

Figure 2. A sample of the patterns having different symmetropy vector values. Black represents +1 and white represents 0 . 
Table 1

Two-Dimensional Walsh Spectra for the Pattern Shown in Figure 2A

\begin{tabular}{|c|c|c|c|c|c|c|c|}
\hline \multicolumn{8}{|c|}{ Component } \\
\hline \multicolumn{2}{|c|}{$\begin{array}{l}\text { Vertically } \\
\text { Symmetric }\end{array}$} & \multicolumn{2}{|c|}{$\begin{array}{c}\text { Horizontally } \\
\text { Symmetric }\end{array}$} & \multicolumn{2}{|c|}{$\begin{array}{l}\text { Centro- } \\
\text { symmetric }\end{array}$} & \multicolumn{2}{|c|}{$\begin{array}{c}\text { Doubly } \\
\text { Symmetric }\end{array}$} \\
\hline$a_{0,1}$ & $1 / 8$ & $a_{1,0}$ & $1 / 8$ & $a_{1,1}$ & 0 & $\mathrm{a}_{0,0}$ & $1 / 2$ \\
\hline $\mathrm{a}_{0,3}$ & 0 & $a_{3,0}$ & $1 / 8$ & $a_{1,3}$ & $-1 / 8$ & $a_{0,2}$ & $-1 / 8$ \\
\hline$a_{2,1}$ & $1 / 8$ & $a_{1,2}$ & 0 & a 3,1 & 0 & $a_{2,0}$ & 0 \\
\hline$a_{2,3}$ & $1 / 4$ & $a_{3,2}$ & $-1 / 4$ & $a_{3,3}$ & $1 / 8$ & $a_{2,2}$ & $1 / 8$ \\
\hline
\end{tabular}

Putting the values given in Table 1 into Equation 2, we obtain

$$
\begin{aligned}
K & =\sum_{m=0}^{3} \sum_{n=0}^{3}\left(a_{m, n}\right)^{2}-\left(a_{0,0}\right)^{2}, \\
& =(1 / 2)-(1 / 4), \\
& =1 / 4 .
\end{aligned}
$$

Then, using Equation 3, $\mathrm{p}_{\mathrm{m}, \mathrm{n}}$ are calculated. Substituting the values for $p_{m, n}$ in Equations 5,6 , 7, and 8, we obtain

$$
P_{1}=3 / 8 \quad P_{2}=3 / 8, \quad P_{3}=1 / 8, \quad P_{4}=1 / 8 .
$$

From Equation 4, we see easily that

$$
\sum_{k=1}^{4} P_{k}=1 .
$$

Hence, we can calculate the symmetropy of pattern A:

$$
\begin{aligned}
S_{4} & =-\sum_{k=1}^{4} P_{k} \log _{2} P_{k}, \\
& =1.81 \text { bits. }
\end{aligned}
$$

Then, in order to obtain the symmetropy vector, the symmetropies of all the $2 \times 2$ patterns within pattern $\mathbf{A}$ are calculated. Putting them at each distinct $2 \times 2$ window position within pattern $A$, we can write

$\begin{array}{ccc}1.58 & 0 & 0 \\ 1.58 & 1.58 & 0 \\ 0 & 0 & 1.58 .\end{array}$

The mean and the standard deviation of these nine symmetropy values are $S_{4}^{1}=.70$ and $\sigma_{4}^{1}=.79$, respectively. Therefore, the symmetropy vector of pattern $A$ is

$$
\begin{aligned}
V_{4}(A) & =\left(S_{4}, S_{4}^{1}, \sigma_{4}^{1}\right), \\
& =(1.81, .70, .79)
\end{aligned}
$$

In the same way, the symmetropy vector of pattern $B$, given in Figure 2, is

$$
V_{4}(B)=(1.0,0,0) \text {. }
$$

\section{Multiple Regression Analyses}

If patterns have the same symmetropy vector, they should be equally good or equally complex. Multiple regression analyses can be used to test the utility of the symmetropy vector as a predictor of pattern goodness and complexity. In the regression analyses, we consider the elements of a symmetropy vector as predictor variables.

\section{Pattern Goodness}

Howe (1980) constructed a set of 60 patterns, each consisting of nine black dots in a $5 \times 5$ square grid, to investigate the effects of partial symmetry in visual patterns on judgment of pattern goodness, immediate memory, and learning. Some examples of the Howe patterns are shown in Figure 3.

In order to relate the symmetropy vector to judged pattern goodness, we use this set of the 60 Howe patterns and their mean goodness values taken from Howe (1980). First, the symmetropy vectors were calculated by a computer program for all of the 60 Howe patterns. Then, using these symmetropy vectors and the mean goodness values, multiple regression analysis was carried out. The computed correlations among the predictor variables and judged pattern goodness are shown in Table 2.

The following regression equation was obtained:

$$
\text { Pattern goodness }=1.90 \mathrm{~S}_{3}+1.38 \sigma_{s}^{2}+1.78 \text {, }
$$

$$
R=.96,
$$

(a)

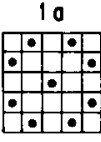

$1.55(1.78)$

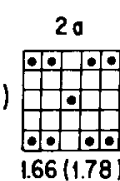

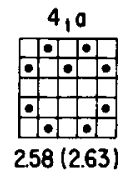
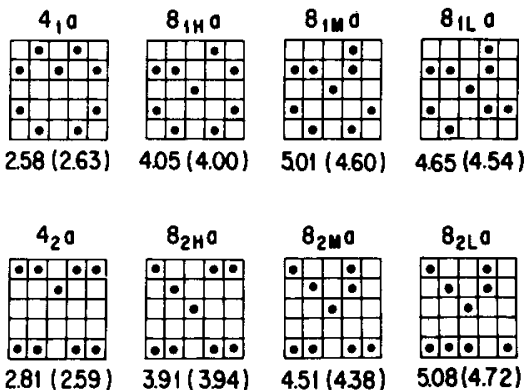

Figure 3. A sample of the Howe patterns (from Howe, 1980). Above each pattern is an identity code. ${ }^{2}$ Under each pattern is the judged goodness value. The number in parentheses following the judged goodness indicates the goodness value predicted by the regression equation obtained in this paper. 
Table 2

Correlations Among Predictor Variables and Judged Pattern Goodness

\begin{tabular}{llrrrr}
\hline Variable & $\mathrm{S}_{\mathrm{s}}$ & $\mathrm{S}_{\mathrm{s}}^{2}$ & $\mathrm{~S}_{\mathrm{s}}^{1}$ & $\sigma_{5}^{2}$ & $\sigma_{5}^{1}$ \\
\hline $\mathrm{S}_{\mathrm{s}}$ & & & & & \\
$\mathrm{S}_{\mathrm{s}}^{2}$ & .12 & & & & \\
$\mathrm{~S}_{3}^{1}$ & .00 & .47 & & & \\
$\sigma_{5}^{2}$ & .49 & -.32 & -.24 & & \\
$\sigma_{s}^{1}$ & .22 & -.19 & -.69 & .29 & \\
Goodness & .95 & .09 & -.03 & .56 & .29 \\
\hline
\end{tabular}

where $\mathbf{R}$ represents the coefficient of multiple correlation. This equation and the high value of the coefficient of multiple correlation indicate that the goodness of each of the 60 Howe patterns is mostly predicted by using only two objective variables, $\mathrm{S}_{3}$ and $o_{5}^{2}$.

\section{Pattern Complexity}

Chipman (1977) used a set of 45 patterns as the stimuli in studying the determinants of judged pattern complexity. The 45 Chipman patterns are $6 \times 6$ matrices of black and white square cells, each with 12 black square cells. Note, however, that the boundary of the matrix is not indicated. Some examples of the Chipman patterns are shown in Figure 4. This time, we used the 45 Chipman patterns and their mean judged complexity values to relate the symmetropy vector to judged pattern complexity. As in the case of pattern goodness stated above, multiple regression analysis was carried out. The computed correlations among the predictor variables and judged pattern complexity are shown in Table 3.

The following two regression equations were obtained:

$$
\begin{aligned}
& \text { Pattern complexity }=7.66 \mathrm{~S}_{8}+20.93 \mathrm{~S}_{8}^{1} \\
& -18.05 o_{\mathrm{B}}^{2}+5.32 \text {, }
\end{aligned}
$$
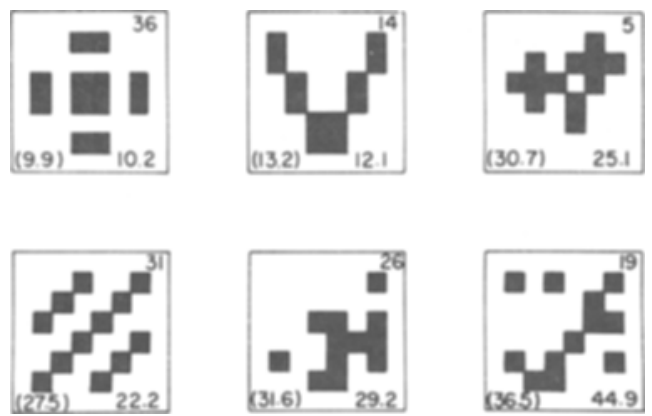

Figure 4. A sample of the Chipman patterns (from Chipman, 1977). At the upper right of each pattern is an identification number. At the lower right is the judged complexity value. The number in parentheses at the lower left indicates the complexity value predicted by the regression equation obtained in this paper.
Table 3

Correlations Among Predictor Variables and Judged Pattern Complexity

\begin{tabular}{lrrrrr}
\hline \multicolumn{1}{c}{ Variable } & \multicolumn{1}{c}{$\mathrm{S}_{\mathbf{8}}$} & \multicolumn{1}{c}{$\mathrm{S}_{\varepsilon}^{2}$} & \multicolumn{1}{c}{$\mathrm{S}_{8}^{1}$} & $\sigma_{8}^{2}$ & $\sigma_{8}^{1}$ \\
\hline $\mathrm{S}_{\mathbf{8}}$ & & & & & \\
$\mathbf{S}_{\mathbf{8}}^{2}$ & .72 & & & & \\
$\mathrm{~S}_{\mathbf{8}}^{1}$ & .47 & .68 & & & \\
$\boldsymbol{\sigma}_{8}^{2}$ & -.66 & -.84 & -.73 & & \\
$\sigma_{8}^{1}$ & .45 & .74 & .90 & -.69 & \\
Complexity & .78 & .77 & .74 & -.80 & .70 \\
\hline
\end{tabular}

$$
\mathrm{R}=.90 \text {, }
$$

and pattern complexity $=9.41 \mathrm{~S}_{8}+29.10 \mathrm{~S}_{8}-7.81$,

$$
\mathbf{R}=.89 \text {, }
$$

where $\mathrm{R}$ represents the coefficient of multiple correlation.

Note that $M$ is equal to 8 , not to 6 , in predictor variables $S_{M}^{k}$ and $o_{M}^{k}$. The reason for this is that multiple regression analyses yielded a higher multiple correlation for $M=8$ than for $M=6$.

The results of multiple regression analyses indicate that the complexity of each of the 45 Chipman patterns is well predicted by using only two objective variables $S_{8}$ and $S_{8}^{1}$, which are the whole symmetropy and the $2 \times 2$ partial symmetropy, respectively.

\section{DISCUSSION}

What are the advantages and disadvantages of using the two-dimensional discrete Walsh transform to develop a measure of visual symmetry? As compared with the discrete Fourier transform and other orthogonal transforms, the Walsh transform has the following advantages: (1) Since the Walsh functions take only the two values +1 and -1 , and the only operations involved are additions and subtractions, the two-dimensional discrete Walsh transform is easier and considerably faster to compute than the discrete Fourier and other orthogonal transforms. (2) Patterns, each consisting of black dots in a square grid, or of a matrix of black and white cells, are used as stimuli in many studies on pattern goodness and pattern complexity. In this case, the two-dimensional discrete Walsh transform provides the same accuracy with fewer terms.

On the other hand, the Walsh transform has the following disadvantages. (1) In general, any pattern must be represented by a $2^{p} \times 2^{q}(p$ and $q$ are positive integers) matrix. (In this paper, the case $p=q$ was chosen.) (2) The two-dimensional discrete Walsh transform is not invariant under each operation of translation, rotation, dilation, and contraction. (3) The 
symmetropy of that pattern which is coincident with one two-dimensional Walsh function other than the correspondence of each gray level equals zero. Therefore, such a pattern must be treated as a singular case. However, (1) is not an essential disadvantage, because any pattern can be represented as a $2^{q} \times 2^{q}$ pattern by use of the method explained in an earlier section. In relation to (2), it should be noted that the Walsh transform has peculiar invariant properties, as stated in an earlier section.

In the first section, it was pointed out that most measures of symmetry proposed in the past (1) are not continuous, while symmetry is a continuum, (2) are not adequate to measure partial symmetry, and (3) do not have enough predictive power.

In the present paper, these problems have been solved by developing a new measure of symmetry, the symmetropy vector. From the definitions of symmetropy and the symmetropy vector, one can easily understand how problems 1 and 2 have been solved. With respect to problem 3, multiple regression analyses of judged pattern goodness and of judged pattern complexity were carried out to test the predictive power of the symmetry vector.

It is important to note that in the 60 Howe patterns used in the regression analysis there was a very high correlation of .95 between whole symmetropy and judged pattern goodness. This indicates that whole symmetropy is a very good representation of the quantitative physical variable underlying perceived pattern goodness.

The regression equations predicting pattern complexity, obtained here for the $\mathbf{4 5}$ Chipman patterns, can be compared with those in Chipman's study (1977). She has obtained the following regression equations for the same set of patterns used here:

Pattern complexity $=.78$ (turns) $-.10(\mathrm{H}-\mathrm{V}$ symmetry)

-.12 (diagonal symmetry)

+43.96 ,

$$
\mathrm{R}=.85 \text {, }
$$

Pattern complexity $=.90($ turns $)-.14(\mathrm{H}-\mathrm{V}$ symmetry $)$

-.13 (diagonal symmetry)

.17 (opposition symmetry)

+64.12 ,

$$
\mathbf{R}=.90 \text {, }
$$

where $\mathbf{R}$ represents the coefficient of multiple correlation. These regression equations may be compared with those obtained in this paper. It should be em- phasized that, in Chipman's equations, the coefficient of multiple correlation is .85 for three variables and .90 for four variables, while in those obtained in this paper, it is .89 for only two variables and .90 for three variables. Furthermore, the more important and fundamental distinction between the two regression models is that, in Chipman's, all the predictor variables are selected out of such geometrical features as the number of turns, the amount of contour, the amount of each type of symmetry, and others, whereas in the present model, the variables are the whole symmetropy, the partial symmetropies, and their standard deviations, which are simpler and more systematic variables.

There are several differences between Chipman's symmetry variables and the symmetropy measures. (1) Since the two-dimensional Walsh functions form a complete orthonormal set, the four symmetry components grouped according to the types of symmetry are linearly independent, whereas Chipman's symmetry variables do not show this mathematically desirable property. (2) By using the linearly independent property of the four symmetry components, it has been possible to define symmetropy as an entropylike measure of symmetry. However, Chipman's symmetry variables were created by simply summing up partial symmetries measured with a moving axis of symmetry. Therefore, although partial symmetropy is created by averaging symmetropy values for all possible locations of a window of a given size, the symmetropy measures themselves are quite different from Chipman's. (3) Chipman used the variable of diagonal symmetry, while symmetropy measures have been developed on the basis of four symmetry types which do not include diagonal symmetry. However, this is not an essentially weak point of the symmetropy measures, since observation windows can be rotated by $45 \mathrm{deg}$, although this has not yet been attempted.

Finally, we examine instances of particularly bad predictions using the symmetropy measures. The four instances of the worst predictions for both the Howe and Chipman patterns are shown in Figure 5. In all of these patterns, the predicted values are smaller than the judged values. That is, all of them are predicted by the symmetropy vector to be simpler or better patterns than is the case by actual judgment.

In the previous studies on pattern goodness and pattern complexity, or its inverse, simplicity, it has long been recognized that a good pattern is simple. However, using linear binary patterns as stimuli, Imai et al. (1976) have reported that judged complexity increases with the number of runs whereas judged goodness does not. Thus, although the two concepts, pattern goodness and pattern simplicity (or complexity), have a fairly high correlation, they are not identical. To make this distinction clear, we examine the results of the multiple regression analyses. Table 2 

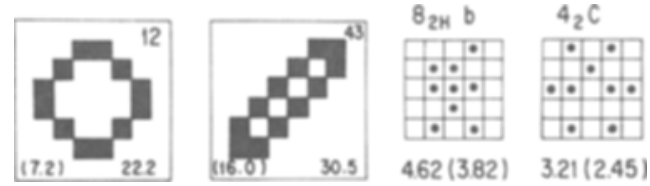

Figure 5. The four instances of the worst predictions using the symmetropy vectors for both the Howe and Chipman patterns.

shows that the correlation between judged pattern goodness and the whole symmetropy is particularly high (.95), as compared with those for the other predictor variables. In addition, Table 3 shows that the correlations between judged pattern complexity and the predictor variables all range from .70 to .80 . These suggest that pattern goodness is a holistic property of a pattern, while pattern complexity (or simplicity) depends on both holistic and partial properties of a pattern.

Finally, in this paper, equal weight is given to the four types of symmetry (i.e., vertical, horizontal, centro-, and double symmetries). However, it has been suggested that vertical symmetry is perceptually more salient than horizontal and rotational symmetry (Corballis \& Roldan, 1975; Palmer \& Hemenway, 1978). Therefore, if we could give appropriate weight to each of the four types of symmetry, symmetropy would become a better objective measure of symmetry, and therefore, a better model of the human perceptual response to visual symmetry.

\section{REFERENCES}

Abramson, N. Information theory and coding. New York: McGraw-Hill, 1963.

Attneave, F. Some informational aspects of visual perception. Psychological Review, 1954, 61, 183-193.

ATtNe ave, F. Symmetry, information and memory for patterns. American Journal of Psychology, 1955, 68, 209-222.

BeAUChAMP, K. G. Walsh functions and their applications. London: Academic Press, 1975.

Blachman, N. M. Sinusoids versus Walsh functions. Proceedings of the IEEE, 1974, 62, No. 3, 346-354.

$\mathrm{C}_{\mathrm{ARL}}, \mathrm{J}$. W. A biologically derived model for image classification utilizing Walsh functions. Proceedings of the 22nd National Aerospace Electronics Convention (NAECON), 1970, 253-259.

Chipman, S. F. Complexity and structure in visual patterns. Journal of Experimental Psychology: General, 1977, 106, 269-301.

Corballis, M. C., \& Roldan, C. E. Detection of symmetry as a function of angular orientation. Journal of Experimental Psychology: Human Perception and Performance, 1975, 1, 221-230.

GARNER, W. R. Uncertainty and structure as psychological concepts. New York: Wiley, 1962.

Garner, W. R., \& Clement, D. E. Goodness of patterns and pattern uncertainty. Journal of Verbal Learning \& Behavior, $1963,2,446-452$.

HaRmuth, H. F. Transmission of information by orthogonal functions. New York: Springer, 1972.

Hocheerg, J., \& McAlister, E. A quantitative approach to figural "goodness." Journal of Experimental Psychology, 1953, 46, 361-364.
Howe, E. S. Effects of partial symmetry, exposure time, and backward masking on judged goodness and reproduction of visual patterns. Quarterly Journal of Experimental Psychology, $1980,32,27-55$.

IмAI, S., Iто, S., \& Iто, T. [Effects of intra-pattern transformation structures and the number of runs upon goodness and complexity judgments of patterns.] Japanese Psychological Review, 1976, 19, 77-94 (in Japanese).

KoffKA, K. Principles of Gestalt psychology. New York: Harcourt Brace Jovanovich, 1935.

Palmen, S. E., \& Hemenway, K. Orientation and symmetry: Effects of multiple, rotational, and near symmetries. Journal of Experimental Psychology: Human Perception and Performance, $1978,4,691-702$.

Szilagyi, P. G., \& Baird, J. C. A quantitative approach to the study of visual symmetry. Perception \& Psychophysics, 1977, 22, 287-292.

WALSH, J. L. A closed set of orthogonal functions. American Journal of Mathematics, 1923, 45, 5-24.

ZUSNE, L. Moments of area and of the perimeter of visual form as predictors of discrimination performance. Journal of Experimental Psychology, 1965, 69, 213-220.

Zusne, L. Measures of symmetry. Perception \& Psychophysics, 1971, 9, 363-366.

\section{APPENDIX}

\section{WALSH FUNCTIONS}

\section{Definition}

There are several different ways of defining and/or representing Walsh functions (Beauchamp, 1975; Harmuth, 1972). In this paper, we adopt the definition shown below (Blachman, 1974) derived from sinusoidal functions, because, although most readers will be unfamiliar with Walsh functions, they will be familiar with Fourier (sine-cosine) functions. In addition, this definition clarifies the relationship between Walsh functions and Fourier functions.

The Walsh function wal $(\mathbf{k}, \mathbf{x})$ of order $\mathbf{k}$ and argument $\mathbf{x}$ can be represented over the interval $0 \leqslant x<1$ as follows:

$$
\begin{aligned}
\operatorname{wal}(k, x) & =\prod_{i=0}^{m-1} \operatorname{sgn}\left[\left(\cos 2^{i} \pi x\right)^{k_{i}}\right], \\
k & =0,1,2, \ldots,
\end{aligned}
$$

where $k_{i}=0$ or 1 and $i=0,1, \ldots, m-1$ are the digits of the binary numeral for the integer $k$ such that

$$
k=\sum_{i=0}^{m-1} k_{i} 2^{i}
$$

and $\mathrm{m}$ is the smallest positive integer such that $2^{\mathrm{m}}>\mathrm{k}$, and the function $\operatorname{sgn}(t)$ is -1 if $t<0$ and +1 if $t>0$. In Equation $\mathrm{A} 1$, when $\left(\cos 2^{\mathrm{i}} \pi \mathrm{x}\right)^{\mathbf{k}_{\mathbf{i}}}=0$, we define

$$
\operatorname{sgn}\left[\left(\cos 2^{i} \pi x\right)^{k_{i}}\right]=\operatorname{sgn}\left\{\left[\cos 2^{i} \pi(x+e)\right]^{k_{i}}\right\},
$$

where $e$ is any real number with $0<e<2^{-m}$. Here, note that, since

$$
\lim _{t \rightarrow+0} t^{t}=1
$$

we define $0^{\circ}=1$. 
The number of sign changes in the interval $0 \leqslant x<1$, of the Walsh function wal $(k, x)$ is called its sequency. It is easily shown that the sequency of wal $(k, x)$ is $k$. The sequency corresponds to the frequency of Fourier sinusoidal functions. It should be noted that some authors define the sequency of wal $(k, x)$ as one-half the number of sign changes in the unit interval.

Superimposition of the first eight Walsh functions and the corresponding Fourier sinusoidal functions is shown in Figure 6. As can be seen in Figure 6, Walsh functions appear to be squared-up versions of Fourier sinusoidal functions. This is not true, since, in general, the sign changes of Walsh functions are not equidistant. The total number of sign changes of Walsh functions and their corresponding sinusoidal functions, however, are the same.

\section{Some Fundamental Properties}

We now present without proof some fundamental properties of Walsh functions that will be needed in the paper. (For detailed explanations, refer to Beauchamp, 1975, and Harmuth, 1972.)

Multiplication. Dyadic addition $h \oplus k$, of nonnegative integers $h$ and $k$, is defined as

$$
\text { where } h=\sum_{i=0} h_{i} 2^{i} \text { and } k=\sum_{i=0}^{f}\left|h_{i}-k_{i}\right| 2^{i} \text {, }
$$

Figure 6. Superimposition of the first eight Walsh functions and the corresponding Fourier sinusoidal functions.
Consequently, the product of the two Walsh functions is given by

$$
\text { wal }(h, x) w a l(k, x)=\text { wal }(h \oplus k, x) \text {, }
$$

where $\oplus$ denotes dyadic addition.

If $h, k<2^{q}, q=0,1,2, \ldots$, then $h \oplus k<2^{q}$. Therefore, from Equation $\mathrm{A} 2$, it is easily shown that the Walsh functions of order $k<2 q, q=0,1,2, \ldots$, form a closed set under multiplication.

Orthonormality.

$$
\begin{aligned}
\int_{0}^{1} \operatorname{wal}(h, x) w a l(k, x) d x & =0, \text { for } h \neq k \\
& =1, \text { for } h=k
\end{aligned}
$$

This means that Walsh functions form an orthonormal set in the interval $0 \leqslant x<1$.

Walsh series. Every function $f(x)$ which is integrable in the Lebesgue sense can be expressed in the interval $0 \leqslant x<1$ as a series of the form

$$
f(x)=\sum_{i=0}^{\infty} a_{i} w a l(i, x),
$$

where the coefficients $a_{i}$ are given by

$$
a_{i}=\int_{0}^{1} f(x) w a l(i, x) d x, \text { for } i=0,1,2, \ldots
$$

As with the Fourier series, Parseval's equivalence holds, and we can write

$$
\sum_{i=0}^{\infty} a_{i}^{2}=\int_{0}^{1} f^{2}(x) d x .
$$

Therefore, Walsh functions form a complete orthonormal set in the interval $0 \leqslant x<1$.

Walsh transform. From Equations $A 3$ and A4, we can define a transform pair just like the Fourier transform,

$$
\begin{array}{r}
f(x)=\sum_{i=0}^{\infty} F(i) w a l(i, x), \\
F(i)=\int_{0}^{1} f(x) w a l(i, x) d x .
\end{array}
$$

Discrete Walsh transform. Let the interval $(0,1)$ be divided into $\mathrm{N}=2^{\mathrm{q}}$ ( $\mathrm{q}$ is a positive integer) with equal subintervals. Next, we define $w_{n}(i)$ as the value of the $n^{\text {th }}$ order Walsh function in the $i^{\text {th }}$ subinterval, where $n, i=0,1,2, \ldots$, $N-1$. Let $a_{i}$ be the sampled value of a function $f(x)$ in the ith subinterval. Thus, the discrete Walsh transform can be defined by

$$
a_{i}=\sum_{n=0}^{N-1} A_{n} w_{n}(i),
$$




$$
A_{n}=\frac{1}{N} \sum_{i=0}^{N-1} a_{i} w_{n}(i),
$$

where $\mathrm{i}, \mathrm{n}=0,1,2, \ldots, \mathrm{N}-1$.

It should be noted that we must make $\mathrm{N}$ equal to a power of 2, that is, $\mathbf{N}=2 \mathrm{q}$, in order that $\mathrm{a}_{\mathrm{i}}$ can be expressed in the form of Equation As.

Symmetries. Replacing $x$ by $1-x$ in Equation $A 1$, we easily see that all of the factors are unchanged except $\operatorname{sgn}(\cos \pi x)$, which changes sign. Since this factor is involved only if $i$ is odd, we obtain wal $(i, 1-x)=(-1)^{i}$ wal $(i, x)$. This means that wal $(i, x)$ has even or odd symmetry about $x=1 / 2$, depending on whether $i$ is even or odd.

\section{Previous Applications}

Walsh functions were introduced by the American mathematician J. L. Walsh in 1923 (Walsh, 1923), but there was little interest in their application until the 1960s, when the advent of digital computers revived interest in Walsh functions. In the last decade, a large number of papers dealing with their applications have appeared in the electrical engineering literature. Many of these are published in the Proceedings of the Symposia on the Applications of Walsh Functions.

Engineers have become interested in studying Walsh functions because (1) they form a complete orthonormal set that can be used as a basis to represent signals in much the same way as Fourier sinusoidal functions, (2) they are particularly suited to applications in digital signal processing using logic circuits or digital computers, because they take only two values, +1 and -1 , and (3) they can be computed more quickly than the equivalent Fourier transforms.

Walsh functions now have many fields of application, including speech processing, pattern recognition, image coding and transmission, data compression, signal processing and multiplexing, switching functions, and logic circuitry.

Here, it is worthwhile to note that the finite discrete Walsh transform has some biological relevance. That is to say, Carl (1970) has shown that interacting neurons are intrinsically capable of performing finite discrete Walsh transform computations. However, whether or not a biological system actually performs such computations remains a matter for conjecture.

To the best of the author's knowledge, no theoretical or experimental results concerning applications of Walsh functions to visual perception have been published.

\section{NOTES}

1. An information source is called a zero-memory source if successive symbols emitted from the source are statistically independent (Abramson, 1963).

2. The first digit of each identity code indicates the number of equivalent patterns by rotation and reflection. The subscript digit indicates that more complex patterns were derived from those having an equivalent subset size of either (a) 1 or (b) 2 . Subscripts $\mathbf{H}$ (high), $\mathbf{M}$ (medium), and L (low) define the degree of partial symmetry (Howe, 1980).

(Manuscript received July 2, 1981; revision accepted for publication May 13, 1982.) 